\title{
Geometry and computation of the Lorenz set
}

Citation for published version (APA):

Vermeulen, A. J., Arin, J., \& Kuipers, J. (2004). Geometry and computation of the Lorenz set. International Game Theory Review, 6, 223-238. https://doi.org/10.1142/S0219198904000162

Document status and date:

Published: 01/01/2004

DOI:

10.1142/S0219198904000162

Document Version:

Publisher's PDF, also known as Version of record

\section{Please check the document version of this publication:}

- A submitted manuscript is the version of the article upon submission and before peer-review. There can be important differences between the submitted version and the official published version of record.

People interested in the research are advised to contact the author for the final version of the publication, or visit the DOI to the publisher's website.

- The final author version and the galley proof are versions of the publication after peer review.

- The final published version features the final layout of the paper including the volume, issue and page numbers.

Link to publication

\footnotetext{
General rights rights.

- You may freely distribute the URL identifying the publication in the public portal. please follow below link for the End User Agreement:

www.umlib.nl/taverne-license

Take down policy

If you believe that this document breaches copyright please contact us at:

repository@maastrichtuniversity.nl

providing details and we will investigate your claim.
}

Copyright and moral rights for the publications made accessible in the public portal are retained by the authors and/or other copyright owners and it is a condition of accessing publications that users recognise and abide by the legal requirements associated with these

- Users may download and print one copy of any publication from the public portal for the purpose of private study or research.

- You may not further distribute the material or use it for any profit-making activity or commercial gain

If the publication is distributed under the terms of Article $25 \mathrm{fa}$ of the Dutch Copyright Act, indicated by the "Taverne" license above, 


\title{
GEOMETRY AND COMPUTATION OF THE LORENZ SET
}

\author{
JAVIER ARIN \\ Departamento de Fundamentos del Análisis Económico \\ Basque Country University, Spain \\ JEROEN KUIPERS \\ Department of Mathematics \\ University Maastricht, The Netherlands \\ DRIES VERMEULEN \\ Department of Quantitative Economics \\ University Maastricht, The Netherlands
}

\begin{abstract}
In this paper we study the geometrical properties of the set of Lorenz allocations of a transferable utility game. We provide procedures to compute a single Lorenz allocation, and even the entire set of Lorenz allocations, that rely solely on linear optimization techniques. These procedures only require a finite number of elementary operations and are therefore easy to implement.
\end{abstract}

Keywords: Game theory; linear programming.

\section{Introduction}

In this paper we deal with what are commonly known as transferable utility games, TU-games for short. In a TU-game there is a finite set of players. With each subset of this set of players (a coalition) a certain real number (the value of the coalition) is associated, usually interpreted as the amount of money this coalition can generate if they operate on their own and do not cooperate with the players outside this coalition. Other interpretations are also possible. This value can for example also be viewed as the claim the coalition has issued on the total amount of money to be divided.

Now the assumption is that the entire set of players decides to cooperate, and thus a certain amount of money, the value of the grand coalition, is generated. The central question addressed in this area of research is how this amount of money should be divided among the players. The second assumption often made in connection with this problem is that there is a division of the total amount of money (usually called an allocation) among the players in such a way that each coalition 
gets at least its value. In other words, if you add the amounts of money allocated to the players of a coalition you end up with an amount of money at least as large as the value of the coalition under consideration. Such an allocation is called a core allocation. A game that has at least one core allocation is called balanced. In this paper we will only consider such balanced TU-games.

\subsection{Egalitarianism}

Egalitarianism is the strife of a community or its representants to spread the total wealth of the community as equally as possible among its members. ${ }^{\text {a }}$ A completely equal division of goods can usually not be obtained when subcommunities have reasonable grounds to lay a claim on part of the wealth to be divided. In the above terminology concerning TU-games this can be expressed as the search for an allocation that divides the value of the grand coalition as equally as possible without violation of the claims of the coalitions. In other words, we want to find a core allocation under which the inequality between the players is as small as possible, given these minimal demands of subcoalitions.

\subsection{Lorenz allocations}

One way to measure the degree of inequality between players given an allocation is via the Lorenz criterion. This criterion works as follows. Given two allocations, first compare the amounts the poorest players get according to the respective allocations. ${ }^{\mathrm{b}}$ Then compare the total amount the two poorest players according to the first allocation get with the total amount the two poorest players get according to the second allocation. Next, make a similar comparison for the three poorest players, et cetera. Now, if it turns out that these amounts according to the first allocation are all larger or equal than they are according to the second one, we say that the first allocation Lorenz dominates the second one. ${ }^{\mathrm{c}}$ A core allocation that is not Lorenz dominated by any other core allocation is called a Lorenz allocation. A Lorenz allocation can thus be viewed as a core allocation from which it is no longer possible to reduce social inequality within the society without violation of the minimal demands of at least one of its subcommunities.

\subsection{Aim of the paper}

In this paper we study the computational aspects connected with the determination of Lorenz allocation. We both address the problem of determining all Lorenz

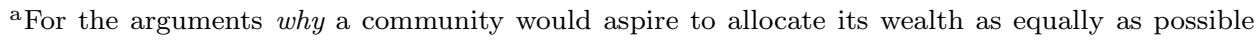
we refer to the discussions in e.g. Rawls (1971) and Dutta and Ray (1989).

${ }^{b}$ Of course the poorest player according to the first allocation need not be the poorest player according to the second. Hence, this comparison may well be between two different players.

${ }^{\mathrm{c}}$ Well, precisely stated, at least one of these amounts should really be strictly larger according to the first allocation then it is according to the second one.
} 
allocations as well as determination of certain special types of Lorenz allocation, such as the lexmax and the lexmin allocations.

\subsection{Organization of the paper}

Section 2 provides the reader with our terminology and some preliminary results. In Sec. 3 we investigate the geometrical properties of the Lorenz set. We prove that the Lorenz set can be decomposed in a finite number of polytopes, called relevant Lorenz polytopes, and that these polytopes constitute a connected polyhedral complex. Similar results are true for the kernel as is shown in Maschler et al. (1979). In Sec. 4 we exploit the results of Sec. 3 to give an algorithm that computes the Lorenz set in a finite number of elementary operations. We also introduce two classes of Lorenz allocations. The algorithm we provide to compute the first class of Lorenz allocations yields relevant Lorenz polytopes. We also provide an algorithm for the second class of Lorenz allocations, which includes the lexmax and the lexmin allocations, and moreover prove that these allocations are extreme points of relevant Lorenz polytopes. In an example we show that not all extreme points of the set of Lorenz allocations can be recovered by this procedure.

\subsection{Notation}

For two elements $k$ and $l$ of a finite set $N$, the directional vector $d_{k l} \in \mathbb{R}^{N}$ is defined by

$$
\left(d_{k l}\right)_{i}:=\left\{\begin{aligned}
1 & \text { if } i=l \\
-1 & \text { if } i=k \\
0 & \text { else. }
\end{aligned}\right.
$$

A subset $F$ of a polytope $P$ is called a face of $P$ if for every $x$ and $y$ in $P$ both $x$ and $y$ are elements of $F$ whenever the vector $\frac{1}{2} x+\frac{1}{2} y$ is an element of $F$. ${ }^{\mathrm{d}}$ Furthermore, the smallest affine subspace ${ }^{\mathrm{e}}$ that contains $P$ is denoted by $\operatorname{ah}(P)$. The relative interior of $P$ is the collection of elements $x$ of $P$ for which for some neighborhood $U$ of $x$ the intersection of $U$ with $\operatorname{ah}(P)$ is a subset of $P{ }^{f}$ A set $X$ in $\mathbb{R}^{n}$ is called connected if it cannot be covered by two disjoint open sets $U$ and $V$ in such a way that both intersections $U \cap X$ and $V \cap X$ are not empty.

A relation $\preceq$ on a set $X$ that satisfies

- (reflexivity) for all $x \in X$ we have $x \preceq x$ and

- (transitivity) for all $x, y, z \in X$ we have $x \preceq z$ whenever both $x \preceq y$ and $y \preceq z$

is called a partial order. It is called a weak order if it also satisfies

\footnotetext{
d Of course there is also a dual definition in terms of linear (in)equalities.

eA subspace $V$ of $\mathbb{R}^{n}$ is called affine if $\lambda x+(1-\lambda) y$ is an element of $V$ for every real number $\lambda$ and elements $x$ and $y$ of $V$.

${ }^{\mathrm{f}}$ Also in this case there is a dual definition in terms of linear (in)equalities.
} 
- (completeness) for all $x, y \in X$ we have at least one of the two relations $x \preceq y$ or $y \preceq x$.

\section{Preliminaries}

A (transferable utility) game is a real-valued function $v: 2^{N} \rightarrow \mathbb{R}$ on the collection $2^{N}$ of subsets of the player set $N:=\{1, \ldots, n\}$ with $v(\emptyset)=0$. Any subset $S$ of the player set $N$ is called a coalition and $v(S)$ is the value of coalition $S$ in the game $v$. We consider the game $v$ to be fixed throughout the paper.

Any real valued vector $x \in \mathbb{R}^{N}$ is called an allocation. The $i$ th coordinate of the vector $x$ represents the payoff given to player $i$. We denote $\sum_{i \in S} x_{i}$ by $x(S)$. The allocation $x$ is efficient if $x(N)=v(N)$. The core $C(v)$ of the game $v$ is defined as the collection of efficient allocations $x$ for which

$$
x(S) \geq v(S)
$$

holds for each coalition $S$. In this paper we will assume that the given game $v$ is balanced, i.e. that its core is not empty.

In order to define the notion of a Lorenz allocation, take an allocation $x$. An order for $x$ is a permutation $\sigma$ of $N$ that orders the coordinates of $x$ in such a way that

$$
x_{\sigma(1)} \leq x_{\sigma(2)} \leq \cdots \leq x_{\sigma(n)} \cdot{ }^{\mathrm{g}}
$$

Now let $y$ be another allocation and let $\tau$ be an order for $y$. We say that $y$ Lorenz dominates $x$ in the weak sense if

$$
\sum_{i=1}^{k} y_{\tau(i)} \geq \sum_{i=1}^{k} x_{\sigma(i)}
$$

for all $k=1, \ldots, n$. Notice that this definition is sound since the above sums do not depend on the particular choices for $\sigma$ and $\tau$. If at least one of the above inequalities is strict, we say that $y$ Lorenz dominates $x$.

Definition 1. A core allocation is called a Lorenz allocation if it is not Lorenz dominated by any other core allocation. The collection of Lorenz allocations is called the Lorenz set in this paper.

It was shown in Arin and Iñarra (1997) that every balanced game has at least one Lorenz allocation.

\section{Geometrical Properties of the Lorenz Set}

The main result of this section is that the Lorenz set is a finite union of polytopes. We will also discuss in what way these polytopes that constitute the Lorenz set

${ }^{\mathrm{g}}$ There may be more than one order in case several players receive the same payoff under the allocation $x$. 
hang together. In particular we will show that they form a connected polyhedral complex. In the next section we will exploit these results for computational ends.

\subsection{Polyhedral structure of the Lorenz set}

First we need some notation. Let $\preceq$ be a weak order on the set $N$ of players of the game $v$. We define the set $A(\preceq)$ by

$$
A(\preceq):=\left\{x \in \mathbb{R}^{n} \mid i \preceq j \text { implies } x_{i} \leq x_{j}\right\} .
$$

Clearly, $A(\preceq)$ is a polyhedral cone. Observe that there are only finitely many different cones of this type, since there are only finitely many weak orders on $N$.

A Lorenz polytope is a set of the form

$$
A(\preceq) \cap F
$$

for some weak order $\preceq$ on $N$ and face $F$ of the core of $v$.

Remarks. Since such a Lorenz polytope is a subset of the bounded set $C(v)$ and both $A(\preceq)$ and $F$ can be given by linear inequalities, a Lorenz polytope is indeed a polytope. Mind though that it is not necessarily a subset of the Lorenz set.

Nevertheless we have the following

Lemma 1. A Lorenz polytope is a subset of the Lorenz set as soon as at least one element of its relative interior is a Lorenz allocation.

Proof. Assume that there is a Lorenz allocation $x$ in the relative interior of a given Lorenz polytope $A(\preceq) \cap F$. Let $y$ be another element of this polytope. We have to show that $y$ is Lorenz. We will do this by contradiction.

So, assume that $y$ is not a Lorenz allocation. Since it is definitely a core allocation, there must be another core allocation, say $y+d$ with $d \neq 0$, that (weakly) Lorenz dominates $y$. Now, since $x$ is in the relative interior of $A(\preceq) \cap F$, we can find an $\eta>0$ as well as a permutation $\sigma$ such that $x+\eta d$ is a core element and $\sigma$ is an order for both $x$ and $x+\eta d$. Then, again since $x$ is an element of the relative interior of $A(\preceq) \cap F, \sigma$ is also an order for $y$. Now let $\tau$ be an order for $y+d$. Then we have for all $k=1, \ldots, n$,

$$
\sum_{i=1}^{k} y_{\sigma(i)}+\sum_{i=1}^{k} d_{\sigma(i)}=\sum_{i=1}^{k}(y+d)_{\sigma(i)} \geq \sum_{i=1}^{k}(y+d)_{\tau(i)} \geq \sum_{i=1}^{k} y_{\sigma(i)} .
$$

The first inequality follows from the fact that $\tau$ is an order for $y+d$ and the second inequality follows from the fact that $y+d$ Lorenz dominates $y$. Now the above inequalities imply that

$$
\sum_{i=1}^{k} d_{\sigma(i)} \geq 0
$$


for all $k=1, \ldots, n$. Thus, it follows that

$$
\sum_{i=1}^{k}(x+\eta d)_{\sigma(i)}=\sum_{i=1}^{k} x_{\sigma(i)}+\eta \sum_{i=1}^{k} d_{\sigma(i)} \geq \sum_{i=1}^{k} x_{\sigma(i)} .
$$

Hence, since $\eta>0$ and $d \neq 0$, this contradicts the assumption that $x$ is a Lorenz allocation.

This lemma by itself is not yet sufficient to conclude that the Lorenz set is the union of a number of Lorenz polytopes. In order to draw that conclusion we also need to know that each Lorenz allocation is an element of the relative interior of some Lorenz polytope. It is not so difficult though to specify such a Lorenz polytope, even for an arbitrary core allocation. In order to do this, let $x$ be a core allocation. Define the weak order $\preceq_{x}$ on $N$ by

$$
i \preceq_{x} j \quad \text { if and only if } x_{i} \leq x_{j} .
$$

Furthermore, let $F(x)$ be the collection of core allocations $y$ for which

$$
y(S)=v(S)
$$

holds as soon as $x(S)=v(S)$. Obviously, $F(x)$ is a face of the core of $v$. Therefore the following definition makes sense.

Definition 2. The set $A\left(\preceq_{x}\right) \cap F(x)$ is called the Lorenz polytope ${ }^{\mathrm{h}}$ associated with the allocation $x$.

It is not difficult to prove that $x$ is an element of the relative interior of its associated Lorenz polytope. This enables us to prove

Theorem 1. The Lorenz set is the union of a (necessarily finite) number of Lorenz polytopes.

Proof. Let $x$ be a Lorenz allocation. As we just remarked, the allocation $x$ is an element of the relative interior of its associated Lorenz polytope. Hence, since each such a Lorenz polytope is therefore automatically contained in the Lorenz set by Lemma 1 , the proof is complete.

\subsection{Polyhedral complexes}

It is possible to say a little bit more about the collection of Lorenz polytopes that constitute the Lorenz set. They form a particularly nice structure that can be expressed as follows.

Definition 3. Let $I$ be a finite set of indices. A (finite) collection

$$
\mathcal{P}:=\left\{P_{i} \mid i \in I\right\}
$$

\footnotetext{
${ }^{\mathrm{h}} \mathrm{It}$ is easy to check that this is indeed a Lorenz polytope.
} 
of polytopes is called a polyhedral complex if for each $i, j \in I$ the intersection $P_{i} \cap P_{j}$ is an element of $\mathcal{P}$ and a face of both $P_{i}$ and $P_{j}$.

Now let us say that a Lorenz polytope is relevant if it is a subset of the Lorenz set. Let $\mathcal{R}$ be the collection of all relevant Lorenz polytopes.

Theorem 2. The collection $\mathcal{R}$ is a polyhedral complex.

Proof. Take two relevant Lorenz polytopes $A\left(\preceq_{1}\right) \cap F$ and $A\left(\preceq_{2}\right) \cap G$. We have to show that the intersection

$$
R:=\left(A\left(\preceq_{1}\right) \cap F\right) \cap\left(A\left(\preceq_{2}\right) \cap G\right)
$$

is a relevant Lorenz polytope and a face of both $A\left(\preceq_{1}\right) \cap F$ and $A\left(\preceq_{2}\right) \cap G$.

A. First we will show that $R$ is a Lorenz polytope. (Once we have that, relevance is immediate.) To this end, define $\preceq$ by writing $i \preceq j$ if there is a sequence $i_{1}, i_{2}, \ldots, i_{k}$ in $N$ such that $i=i_{1}, i_{k}=j$ and

$$
i_{1} \preceq \text { ? } i_{2} \preceq \text { ? } \cdots \preceq \text { ? } i_{k}
$$

where each question mark indicates either index 1 or index $2 .{ }^{i}$ It is straightforward to check that $\preceq$ is indeed a weak order. Furthermore, $H:=F \cap G$ is clearly a face of the core of $v$. We will show that $R$ equals $A(\preceq) \cap H$. Since the reverse inclusion is trivial, we will only show that $R$ is a subset of $A(\preceq) \cap H$.

So, take an allocation $x$ in $R$. Since this is clearly an element of $H$, we only need to show that it is included in $A(\preceq)$. To this end, assume that $i \preceq j$. We have to show that $x_{i} \leq x_{j}$. However, since $i \preceq j$, there is a sequence $i_{1}, i_{2}, \ldots, i_{k}$ in $N$ such that $i=i_{1}, i_{k}=j$ and

$$
i_{1} \preceq_{?} i_{2} \preceq_{?} \cdots \preceq_{?} i_{k}
$$

where the question mark indicates either index 1 or index 2. Now, since $x$ is an element of both $A\left(\preceq_{1}\right)$ and $A\left(\preceq_{2}\right)$, this implies that

$$
x_{1}=x_{i_{1}} \leq x_{i_{2}} \leq \cdots \leq x_{i_{k}}=x_{j}
$$

which concludes this part of the proof.

B. By symmetry it is sufficient to show that $R$ is a face of $A\left(\preceq_{1}\right) \cap F$. To this end, take two allocations $x$ and $y$ in $A\left(\preceq_{1}\right) \cap F$ and assume that $z:=\frac{1}{2} x+\frac{1}{2} y$ is an element of $R$. We have to show that $x$ and $y$ are elements of $R$. Since they are elements of $A\left(\preceq_{1}\right)$ by the way we chose them, we only have to show that they are elements of $A\left(\preceq_{2}\right)$ and $H$.

B1. In order to show that $x$ and $y$ are elements of $H$, notice that $x$ and $y$ are elements of the core of $v$. So, since $z=\frac{1}{2} x+\frac{1}{2} y$ is in particular an element of the face $H$ of the core of $v, x$ and $y$ must also be elements of $H$.

${ }^{\mathrm{i}}$ Meaning that both orders $\preceq_{1}$ and $\preceq_{2}$ are allowed to be used in the same displayed sequence of inequalities. 
B2. The only thing left to show is that $x$ (and then also $y$ ) is an element of $A\left(\preceq_{2}\right)$. So, take $i$ and $j$ with $i \preceq_{2} j$. We have to show that $x_{i} \leq x_{j}$. We will do this by contradiction. So, assume that $x_{i}>x_{j}$. Then, since $x$ is an element of $A\left(\preceq_{1}\right)$ and $\preceq_{1}$ is complete, we know that $i \succeq_{1} j$. So, since $y$ is also an element of $A\left(\preceq_{1}\right)$, we know that $y_{i} \geq y_{j}$. Hence,

$$
z_{i}=\frac{1}{2} x_{i}+\frac{1}{2} y_{i}>\frac{1}{2} x_{j}+\frac{1}{2} y_{j}=z_{j} .
$$

Thus we get that $z_{i}>z_{j}$. This though contradicts the fact that $z$ is an element of $R \subset A\left(\preceq_{2}\right)$ and $i \preceq_{2} j$.

Remarks. A relevant Lorenz polytope is called maximal if it is not properly contained in any other relevant Lorenz polytope. Obviously, we now have that the Lorenz set is the union of maximal Lorenz polytopes. Note that it follows from the previous Theorem that every relevant Lorenz polytope is a face of a maximal relevant Lorenz polytope.

\subsection{Connectedness}

Finally in this section we will show that the Lorenz set is connected. First we need some notation.

Definition 4. Let $G$ be a closed set of Lorenz allocations. The set of core allocations that are weakly Lorenz dominated by some allocation in $G$ is denoted by $D(G)$.

Notice that $G$ is automatically a subset of $D(G)$. Furthermore we have

Lemma 2. $D(G)$ is a closed set.

Proof. Let $\left(x^{t}\right)_{t \in \mathbb{N}}$ be a sequence of allocations in $D(G)$ converging to some limit $x$. We have to show that $x$ is an element of $D(G)$.

Since $x^{t}$ is an element of $D(G)$, we can take an allocation $y^{t}$ in $G$ such that $y^{t}$ weakly Lorenz dominates $x^{t}$. Furthermore, since $G$ is assumed to be a closed subset of the core of $v$, it is compact. So, we can assume w.l.o.g. that the sequence $\left(y^{t}\right)_{k \in \mathbb{N}}$ converges to some allocation $y$ in $G$. It is also clear that $x$ is a core element, since it is the limit of a sequence of core elements. So, it is sufficient to show that $y$ weakly Lorenz dominates $x$.

To this end, notice that, by taking subsequences, we can guarantee that there are two permutations $\sigma$ and $\tau$ of $N$ such that $\sigma$ is an order for each $x^{t}$ and $\tau$ is an order for each $y^{t}$. So, since each $y^{t}$ weakly Lorenz dominates the corresponding $x^{t}$, we know that

$$
\sum_{i=1}^{k} y_{\tau(i)}^{t} \geq \sum_{i=1}^{k} x_{\sigma(i)}^{t}
$$


for all $k=1, \ldots, n$. Since these inequalities remain true when we take limits, and $\sigma$ and $\tau$ are still orders for the limits $x$ and $y$ respectively, we get that $y$ weakly Lorenz dominates $x$.

Using this result we can show

Theorem 3. The Lorenz set is connected.

Proof. We will show this by contradiction. So, assume that the Lorenz set of $v$ is not connected. Then, since the Lorenz set is closed, it can be divided into two non-empty, closed and mutually disjoint sets, say $F$ and $G$. First notice that $D(F)$ and $D(G)$ are closed by Lemma 2 . They are also not empty, since they include the non-empty sets $F$ and $G$, respectively. So, since - by Lemma 4 in the Appendix $D(F)$ and $D(G)$ cover the connected set $C(v), Z:=D(F) \cap D(G)$ is not empty. Since $Z$ is also clearly compact, it follows that $Z$ contains at least one element that is not Lorenz dominated by any other element of $Z$. Let $z \in Z$ be such a point. Then, since $z$ is an element of $D(F)$, we can choose an allocation $x$ in $F$ that weakly Lorenz dominates $z$. Similarly we can choose an allocation $y$ in $G$ that weakly Lorenz dominates $z$. So, since $D(F)$ and $D(G)$ form a closed cover of the core of $v$, there must be an element of the line segment

$$
\{\mu x+(1-\mu) y \mid 0 \leq \mu \leq 1\},
$$

say $w:=\mu^{*} x+\left(1-\mu^{*}\right) y$, that is an element of $Z=D(F) \cap D(G)$. However, $x$ is not an element of $G$ and $y$ is not an element of $F$, since $F$ and $G$ are disjoint. So, $0<$ $\mu^{*}<1$, which implies that $w$ (strictly!) Lorenz dominates $z$. This contradicts the fact that $z$ is not Lorenz dominated by any other element of $Z$. Hence, the Lorenz set is connected.

\section{Computational Aspects}

In this section we will discuss several methods that can be used to calculate Lorenz allocations. First we will explain how the entire Lorenz set may be computed using the results from the previous section. Secondly we will present some algorithms for the computation of a single Lorenz allocation.

\subsection{Computation of all Lorenz allocations}

The results from the previous section enable us to compute the Lorenz set in a finite number of steps. To see this, notice that by Theorem 1 computation of the Lorenz set boils down to the question whether we can determine the collection $\mathcal{R}$ of all relevant Lorenz polytopes.

Now first of all, notice that it is not difficult to list all Lorenz polytopes. The sets $A(\preceq)$ are given by a finite number of linear inequalities after all (depending on the specific choice of $\preceq$ ) and the faces of the core of $v$ are also available in the form 
of a finite number of linear inequalities. The difficult part is to determine whether or not the intersection $A(\preceq) \cap F$ of a given pair of such sets is relevant. For this, we first need to compute a specific element, say $z$, of the relative interior of $A(\preceq) \cap F$. This can be done using standard linear optimization techniques. Then, by Lemma 1 it is sufficient to check whether $z$ is a Lorenz allocation. This can be done as follows. Let $\sigma$ be an order for $z$. Define

$$
\alpha_{k l}(z):=\frac{1}{2}\left(z_{\sigma(k)}-z_{\sigma(l)}\right)
$$

for every $k$ and $l$ with $l<k$. Furthermore, given a vector $\lambda=\left(\lambda_{k l}\right)_{l<k}$, define, for the sake of brevity, the vector $d(\lambda, z)$ in $\mathbb{R}^{n}$ by

$$
d(\lambda, z):=\sum_{l<k} \lambda_{k l} \alpha_{k l}(z) d_{\sigma(k) \sigma(l)}^{\mathrm{j}}
$$

Consider the following linear program $L P(z)$.

$$
\begin{aligned}
& \operatorname{maximize} \sum_{l<k} \lambda_{k l} \alpha_{k l}(z) \\
& \text { subject to }\left\{\begin{array}{l}
x+d(\lambda, z) \in C(v) \\
\sum_{l<k} \lambda_{k l} \leq 1 \\
\lambda
\end{array}\right.
\end{aligned}
$$

Now let $\mu$ be any optimal solution of $L P(z)$. It is shown in Arin et al. (2000) that $z$ is a Lorenz allocation if and only if $d(\mu, z)=0$. Thus, we can also use standard linear optimization techniques to check whether or not the allocation $z$ in the relative interior of $A(\preceq) \cap F$ is Lorenz.

\subsection{Computation of one Lorenz allocation}

In this section we will provide two techniques for the computation of Lorenz allocations. The first technique is based on the fact that maximization of certain realvalued functions over the core of the game $v$ yields elements of the Lorenz set $v$. The second technique is based on lexicographic maximization of the Lorenz curve over the core of $v$.

\subsection{Real-valued maximization}

In order to define the class of functions we will consider, let $\beta=\left(\beta_{1}, \ldots, \beta_{n}\right)$ be a vector with $\beta_{i}>0$ for all $i$. The $\beta$-Lorenz function $l_{\beta}$ is defined as follows. Let $x$ be any allocation. Let $\sigma(x)$ be an order for $x$. Then

$$
l_{\beta}(x):=\sum_{k=1}^{n} \beta_{k} \sum_{i=1}^{k} x_{\sigma(x)(i)} .
$$

${ }^{\mathrm{j}}$ Notice that this definition does not depend on the specific choice of the order $\sigma$ for $z$. 
Notice that this definition is sound, since it does not depend on the particular choice of $\sigma(x)$.

We are interested in the collection $\mathcal{O}(\beta)$ of allocations $x$ in the core of $v$ for which

$$
l_{\beta}(x) \geq l_{\beta}(y)
$$

for any core allocation $y$ of $v$. First of all, notice that $\mathcal{O}(\beta)$ is not empty and compact, since $l_{\beta}$ is a continuous function and the core of $v$ is compact. Furthermore we have that $l_{\beta}(x)>l_{\beta}(y)$ if $x$ Lorenz dominates $y$. So, $\mathcal{O}(\beta)$ is a subset of the Lorenz set. We will show that it is even a (automatically relevant) Lorenz polytope.

Theorem 4. The set $\mathcal{O}(\beta)$ is a Lorenz polytope.

Proof. A. First we will show that $\mathcal{O}(\beta)$ is convex. To this end, notice that the $\beta$-Lorenz function satisfies the following two properties.

- $l_{\beta}(\alpha x)=\alpha l_{\beta}(x)$ for all $\alpha \geq 0$ and all $x \in \mathbb{R}^{n}$.

- $l_{\beta}(x+y) \geq l_{\beta}(x)+l_{\beta}(y)$ for all $x, y \in \mathbb{R}^{n}$. Moreover, if there are indices $i$ and $j$ such that $x_{i}<x_{j}$ and $y_{i}>y_{j}$, then $l_{\beta}(x+y)>l_{\beta}(x)+l_{\beta}(y)$.

Hence, if $x$ and $y$ both maximize the $\beta$-Lorenz function over the core of $v$, then so does $\lambda x+(1-\lambda) y$ for all $\lambda \in[0,1]$. It follows that $\mathcal{O}(\beta)$ is a convex set.

B. In this part we will show that $\mathcal{O}(\beta)$ is a Lorenz polytope. Since $\mathcal{O}(\beta)$ is convex, its relative interior is not empty. Hence, we can choose an allocation $z$ in the relative interior of $\mathcal{O}(\beta)$. Let $A\left(\preceq_{z}\right) \cap F(z)$ be its associated Lorenz polytope. We will show that this Lorenz polytope is equal to $\mathcal{O}(\beta)$.

Since the restriction of $l_{\beta}$ to this polytope is a linear function that attains its maximum in the allocation $z$ in the relative interior of the polytope $A\left(\preceq_{z}\right) \cap F(z), l_{\beta}$ must be constant on this polytope. Hence, this polytope must be a subset of $\mathcal{O}(\beta)$.

In order to show the converse inclusion, take an allocation $x$ in $\mathcal{O}(\beta)$. We have to show that $x$ is an element of the above Lorenz polytope $A\left(\preceq_{z}\right) \cap F(z)$. Since $z$ is in the relative interior of $\mathcal{O}(\beta)$, there exists a real number $\varepsilon>0$ such that $z+\varepsilon(z-x)$ is still an element of $\mathcal{O}(\beta)$. This though implies in particular that $x$ and $z+\varepsilon(z-x)$ are core elements, while $z$ is an element of the face $F(z)$ of the core of $v$. So, $x$ must be an element of $F(z)$ as well.

Finally we need to show that $x$ is an element of $A\left(\preceq_{z}\right)$. We will show this by contradiction. So, assume that $i \preceq_{z} j$ and $x_{i}>x_{j}$. Then, since $x$ is an element of $\mathcal{O}(\beta)$ and $z$ is an element of the relative interior of $\mathcal{O}(\beta)$, we know that there exists an $\varepsilon>0$ such that $y:=(1+\varepsilon) z-\varepsilon x$ is still an element of $\mathcal{O}(\beta)$. This though, since $y_{i}<y_{j}$, implies that $l_{\beta}\left(\frac{1}{2} x+\frac{1}{2} y\right)$ is strictly larger than $\frac{1}{2} l_{\beta}(x)+\frac{1}{2} l_{\beta}(y)$. This contradicts the assumption that $x$ and $y$ are elements of the set $\mathcal{O}(\beta)$. 
Remarks. One way to compute an element of $\mathcal{O}(\beta)$ works as follows. Given a permutation $\sigma$, consider the set

$$
C(\sigma):=\left\{x \in C(v) \mid x_{\sigma(1)} \leq \cdots \leq x_{\sigma(n)}\right\}
$$

of core allocations for whom $\sigma$ is an order. Clearly this set is bounded and given by a number of linear inequalities. So, since $l_{\beta}$ coincides with the linear function

$$
x \mapsto \sum_{k=1}^{n} \beta_{k} \sum_{i=1}^{k} x_{\sigma(i)}
$$

on $C(\sigma)$, we can use linear optimization techniques to compute a (core) allocation $x(\sigma)$ that maximizes $l_{\beta}$ over this set $C(\sigma) .{ }^{\mathrm{k}}$ Now evaluate $l_{\beta}$ in each $x(\sigma)$ we can thus construct. Choose an allocation $x(\tau)$ such that

$$
l_{\beta}(x(\tau)) \geq l_{\beta}(x(\sigma))
$$

for all other allocations $x(\sigma)$. This allocation $x(\tau)$ must necessarily be an element of $\mathcal{O}(\beta)$ (and hence a Lorenz allocation). Moreover, if we use an optimization technique that automatically generates interior solutions to the linear programs to be solved, $\mathcal{O}(\beta)$ will be equal to the Lorenz polytope associated with $x(\tau)$.

\subsection{Lexicographic maximization}

The second technique to generate Lorenz allocations works as follows. Let $x$ be an allocation and let $\sigma(x)$ be an order for $x$. The Lorenz curve of $x$ is the vector

$$
\theta(x):=\left(x_{\sigma(x)(1)}, x_{\sigma(x)(1)}+x_{\sigma(x)(2)}, \ldots, \sum_{i=1}^{n} x_{\sigma(x)(i)}\right) .
$$

Now it is easy to verify that a core allocation $x$ for which $\theta(x)$ is lexicographically greater than $\theta(y)$ for any other core allocation $y^{1}$ is automatically a Lorenz allocation. Thus, if we can find a core allocation where $\theta$ attains its lexicographical maximum over the core, we have found a Lorenz allocation.

To pursue this idea a little bit further, let $\pi$ be some permutation of the elements of $N$, and define the vector function $\theta^{\pi}$ by

$$
\theta^{\pi}(x)_{k}:=\theta(x)_{\pi(k)}
$$

Then also any core allocation $x$ for which $\theta^{\pi}(x)$ is lexicographically greater than $\theta^{\pi}(y)$ for any core allocation $y$ will be a Lorenz allocation.

Remarks. Lexicographic maximization of the vector $\theta^{\pi}$ leads to a unique solution. This is seen as follows. Suppose that $x$ and $y$ both maximize $\theta^{\pi}$. Then

${ }^{\mathrm{k}}$ Provided of course that this set is not empty. However, at least one such set will not be empty, because the game $v$ is assumed to be balanced.

${ }^{1}$ We say that $\theta(x)$ is lexicographically greater than $\theta(y)$ if $\theta(x) \neq \theta(y)$ and $\theta(x)_{i}>\theta(y)_{i}$ for the first coordinate $i$ for which $\theta(x)$ and $\theta(y)$ are different. 
$\sum_{i=1}^{k} x_{\sigma(x)(i)}=\sum_{i=1}^{k} y_{\sigma(y)(i)}$ for $k=1, \ldots, n$, and it follows that $x_{\sigma(x)(k)}=y_{\sigma(y)(k)}$ for $k=1, \ldots, n$. Hence, the coordinates of $x$ are the same numbers as the coordinates of $y$, but they are possibly permuted. However, if they are permuted, then one easily shows that $\frac{1}{2} x+\frac{1}{2} y$ Lorenz dominates both $x$ and $y$, a contradiction. We conclude that $x=y$.

Given a permutation $\pi$, the allocation that lexicographically maximizes the function $\theta^{\pi}$ over the core of $v$ is denoted by $z(\pi)$.

The allocation $z(\pi)$ can be computed as follows. For $1 \leq k \leq n$, define the vector $c_{k}$ in $\mathbb{R}^{n}$ by

$$
c_{k i}:= \begin{cases}1 & \text { if } i \leq k \\ 0 & \text { else. }\end{cases}
$$

Now take a (for the moment) fixed permutation $\sigma$. First write $G_{0}(\sigma):=C(\sigma, v)$. Now for $1 \leq k \leq n$ we can inductively define $G_{k}(\sigma)$ as the set of allocations $x$ in $G_{k-1}(\sigma)$ for which

$$
\left\langle c_{\pi(k)}, x\right\rangle \geq\left\langle c_{\pi(k)}, y\right\rangle
$$

for all other allocations $y$ in $G_{k-1}(\sigma)$. All these sets can be computed in terms of finite systems of linear (in)equalities and, moreover, $G_{n}(\sigma)$ is a one-point set. ${ }^{\mathrm{m}}$ Denote the unique allocation in $G_{n}(\sigma)$ by $y(\sigma)$. Now for each such allocation we can compute its "permuted" Lorenz curve

$$
\left(\left\langle c_{\pi(1)}, y(\sigma)\right\rangle, \ldots,\left\langle c_{\pi(n)}, y(\sigma)\right\rangle\right)
$$

The allocation $y(\tau)$ whose permuted Lorenz curve is lexicographically bigger than the permuted Lorenz curve of any other $y(\sigma)$ will be equal to $z(\pi)$.

Intuitively, lexicographic maximization of $\theta^{\pi}$ corresponds to the maximization of a $\beta$-Lorenz function, where $\beta_{\pi(i+1)} \gg \beta_{\pi(i)}$ for $i=1, \ldots, n-1$. According to this intuition one expects that the set of solutions that maximize $\theta^{\pi}$ is a Lorenz polytope, and since this set consists solely of the allocation $z(\pi)$, this allocation should be an extreme point of a maximal Lorenz polytope by Theorem 2 . The following theorem shows that this intuition is correct.

Theorem 5. The allocation $z(\pi)$ is an extreme point of a maximal Lorenz polytope.

Proof. It suffices to prove that $\{z(\pi)\}$ equals the Lorenz polytope associated with $z(\pi)$. Recall that $z(\pi)$ lies in the relative interior of its associated Lorenz polytope $A\left(\preceq_{z(\pi)}\right) \cap F(z(\pi))$. Now suppose that $z(\pi)$ is not the only allocation in $A\left(\preceq_{z(\pi)}\right.$ ) $\cap F(z(\pi))$. Then there must be a non-degenerate line-segment $[a, b] \subset A\left(\preceq_{z(\pi)}\right) \cap F(z(\pi))$, such that $x$ lies in the relative interior of $[a, b]$. Observe that each coordinate of $\theta^{\pi}$ is linear on $A\left(\preceq_{z(\pi)}\right)$. Hence, the first coordinate must be constant on the line segment $[a, b]$, since otherwise either $\theta^{\pi}(a)$ or $\theta^{\pi}(b)$ would

${ }^{\mathrm{m}}$ Again, the condition is that we started with a non-empty set $C(\sigma)$. 
be lexicographically greater than $\theta^{\pi}(z(\pi))$. Knowing that the first coordinate is constant, we can repeat the same argument to show that also the second coordinate of $\theta^{\pi}$ is constant on $[a, b]$, etc. It follows that the function $\theta^{\pi}$ is constant on $[a, b]$. This contradicts the fact that there is a unique vector that maximizes $\theta^{\pi}$ lexicographically.

An obvious question now is: is every extreme point of a Lorenz polytope the lexicographic maximum of some vector $\theta^{\pi}$ ? The answer is negative. Let $v$ be a five-person game where $v(N)=11, v(1234)=10.5, v(12)=v(13)=7$, $v(123)=9.5, v(15)=3.8$ and $v(S)=0$ otherwise. Lexicographic maximization of the vector $\theta^{(1,2,3,4,5)}$ gives us the point $a=(4.5,2.5,2.5,1,0.5)$. Maximization of $\theta^{(5,4,3,2,1)}$ gives $b=(3.5,3.5,3.5,0.2,0.3)$, and maximization of $\theta^{(1,5,4,3,2)}$ gives $c=(4,3,3,0.5,0.5)$. For all other permutations $\pi$ the lexicographic maximum of $\theta^{\pi}$ coincides with $a, b$ or $c$. One can check however that also $d=\frac{1}{15}(53,52,52,4,4)$ is an element of the Lorenz set, and that it is even an extreme point of a maximal Lorenz polytope. In fact, the maximal relevant Lorenz polytopes of the game $v$ are given by the three line segments $[a, c],[d, b]$ and $[c, d]$.

\section{Appendix}

In this section we will prove a Lemma needed in this paper. We will use the Lemma of Zorn, a statement equivalent with the Axiom of Choice. First we need some notation.

Let $X$ be a non-empty set an let $\preceq$ be a partial order on $X$. So, $\preceq$ is assumed to be reflexive and transitive.

A subset $C$ of $X$ is called a chain if for any two elements $x$ and $y$ of $C$ we have at least one of the two inequalities $x \preceq y$ and $y \preceq x$.

A chain $C$ of $X$ is said to have an upper bound if there exists an element $a$ of $X$ such that $x \preceq a$ for all $x$ in $C$.

An element $a$ of $X$ is called maximal if for any $x$ in $X$ the inequality $a \preceq x$ only holds if $x \preceq a$ holds as well. In words, there is no element $x$ that is "really bigger" than $a$.

Now the Axiom of Choice is equivalent with the following:

Lemma 3. (Zorn) Suppose that every chain of $X$ has an upper bound. Then $X$ has a maximal element.

We can use this axiom in the following way. On the collection of core allocations of $v$ we can define the partial order $\preceq_{l}{ }^{\mathrm{n}}$ by writing $x \preceq_{l} y$ if the core allocation $y$ weakly Lorenz dominates the core allocation $x$. We will apply the Lemma of Zorn to this partial order in order to prove:

\footnotetext{
${ }^{\mathrm{n}}$ It is very easy to check that $\preceq_{l}$ is indeed a partial order.
} 
Lemma 4. Every core allocation is weakly Lorenz dominated by a Lorenz allocation.

Proof. Let $x$ be a core allocation. Consider the set

$$
X:=\left\{y \in C(v) \mid x \preceq_{l} y\right\}
$$

of core allocations that weakly Lorenz dominate $x$. Since $X$ contains $x$ this is obviously not an empty set and $\preceq_{l}$ is a partial order on it. We will first check that within $X$ the condition of the Lemma of Zorn is satisfied.

To this end, let $C$ be a chain in $X$. Let $\sigma$ be a permutation of $N$. Let $C(\sigma)$ be the collection of elements in $C$ for which $\sigma$ is an order. ${ }^{\circ}$ Next, for $i \in N$, define the allocation $x(\sigma)$ by

$$
x(\sigma)_{\sigma(k)}:=\sup _{y \in C(\sigma)} \sum_{i=1}^{k} y_{\sigma(i)}-\sup _{y \in C(\sigma)} \sum_{i=1}^{k-1} y_{\sigma(i)} \cdot{ }^{\mathrm{p}}
$$

Since $C(\sigma)$ is a chain in the closed set $X$, it is easily verified that $x(\sigma)$ is also an element of $X$. Moreover, it is an upper bound on $C(\sigma)$.

Now recall that $C$ is a chain. So, for any two permutations $\sigma$ and $\tau$ for which $C(\sigma)$ and $C(\tau)$ are not empty, we have at least one of the two inequalities $x(\sigma) \preceq_{l}$ $x(\tau)$ or $x(\tau) \preceq_{l} x(\sigma)$. This implies that there is a permutation $\rho$ such that $x(\rho)$ weakly Lorenz dominates all other allocations $x(\sigma)$. This though implies that $x(\rho)$ is an upper bound for the entire chain $C$.

Now we can apply the Lemma of Zorn and we get a maximal element of $X$, say $z$. This allocation weakly Lorenz dominates $x$ by definition of $X$. Our claim is that it is a Lorenz allocation. Suppose that $z$ is not a Lorenz allocation. Since it is a core allocation, there must be another core allocation $w$ that Lorenz dominates $z$. That is, $z \preceq_{l} w$, but not $w \preceq_{l} z$. However, $z \preceq_{l} w$ implies that $w$ is an element of $X$ by the transitivity of $\preceq_{l}$. Then though the fact that $w \preceq_{l} z$ does not hold contradicts the assumption that $z$ is a maximal element of $X$.

\section{Acknowledgements}

The authors would like to thank The Basque Country University for financial support, grant UPV 035.321-HB149/96, and also the financial support of Department of Education of Basque government.

\section{References}

Arin, J. and Iñarra, E. [1997] On Consistency and Egalitarianism: The Egalitarian Set, SEEDS 163, Departamento de Fundamentos del Análisis Económico, Basque Country University. 
Arin, J., Kuipers, J. and Vermeulen, D. [2000] Negotiation Processes with Egalitarian Outcomes, Departamento de Fundamentos del Análisis Económico, Basque Country University.

Dutta, B. [1990] "The egalitarian solution and reduced game properties in convex games", International Journal of Game Theory 19, 153-169.

Dutta, B. and Ray, D. [1989] "A concept of egalitarianism under participation constraints", Econometrica 57, 615-635.

Hardy, G. H., Littlewood, J. E. and Pólya, G. [1952] Inequalities, 2nd edition (Cambridge University Press, Cambridge, USA).

Maschler, M., Peleg, B. and Shapley, L. S. [1979] "Geometric properties of the kernel, nucleolus, and related solution concepts", Mathematics of Operations Research 4, 303-338.

Rawls, J. [1971] A Theory of Justice (Harvard University Press, Cambridge, Massachusetts, USA). 